\title{
Estado nutrimental del agroecosistema rosa (Rosa spp.) en la ladera este del Iztaccíhuatl
}

\section{Nutritional status of a rose agroecosystem (Rosa spp.) on the eastern slope of the volcano Iztaccihuatl}

\author{
Steinger Cortés Jiménez ${ }^{1}$, Jorge D. Etchevers Barra ${ }^{2}$, \\ Claudia M. I. Hidalgo Moreno ${ }^{2:}$ y Hermilio Navarro Garza ${ }^{2}$
}

\footnotetext{
${ }^{1}$ Ex-estudiante de la Maestría en Ciencias en Edafología, Colegio de Postgraduados, ${ }^{2}$ Colegio de Postgraduados, Campus Montecillo. Carretera MéxicoTexcoco km. 36.5. 56230 Montecillo, Texcoco, Edo. de México, México.

*Autora responsable (hidalgo@colpos.mx)
}

\begin{abstract}
RESUMEN
Aproximadamente 3000 ha se encuentran plantadas con rosas (Rosa spp.) en el estado de Puebla. El valor estimado generado por esta actividad es 625 millones de pesos y permite incorporar a la mujer a la economía regional. El uso de fertilización nitrogenada en rosa de corte, mayor a $7000 \mathrm{~kg} \mathrm{ha}^{-1}$, es excesiva y afecta la salud del suelo y la economía de los productores. Los objetivos del trabajo fueron: (1) evaluar el estado actual de la fertilidad de los suelos en la zona de producción de rosa del DDR 05 San Martín Texmelucan y (2) estudiar el efecto del aporte de tres fuentes de materia orgánica en la producción, calidad y estado nutrimental de tres variedades de rosas, así como en la condición física de los suelos. Para ello se evaluaron algunas propiedades químicas en muestras de suelo de 112 invernaderos localizados en cuatro unidades edafoclimáticas. Se usaron tres tipos de materia orgánica (MO): compost comercial, estiércol de bovino y gallinaza y tres variedades de rosa: Anastasia, Selena y Latin. El diseño fue completamente al azar modificado y se realizaron análisis de varianza y comparaciones de medias. Los suelos presentaron condiciones de fertilidad contrastantes. La gallinaza aumentó el rendimiento en la variedad Anastasia y el compost en la variedad Selena. La calidad de las tres variedades de rosas aumentó con el aporte de las tres fuentes de MO a los tres meses, pero disminuyó a los nueve meses. Las tres fuentes de MO aportaron niveles similares de macroelementos a la planta y mejoraron la condición física del suelo al aumentar el porcentaje de macroagregados en todos los tratamientos. El mayor porcentaje de macroagregados se obtuvo por la adición de compost en los suelos
\end{abstract}

Cita recomendada:

Cortés Jiménez, S., J. D. Etchevers Barra, C. M. I. Hidalgo Moreno y H. Navarro Garza. 2017. Estado nutrimental del agroecosistema rosa (Rosa spp.) en la ladera este del Iztaccihual. Terra Latinoamericana35: 237-246. con las variedades Anastasia y Selena. Los suelos de los invernaderos del agrosistema rosa (Rosa spp.) presentaron características químicas y condiciones de fertilidad contrastantes, producto de una variabilidad edafoclimática. Las fuentes de materia orgánica usadas tuvieron efecto en el rendimiento y la calidad de las rosas; así como en el porcentaje de macroagregados lo que mejoró la condición física del suelo.

Palabras clave: ornamentales, flor de corte, salud del suelo.

\section{SUMMARY}

Approximately 3600 hectares are planted with roses (Rosa spp.) in the state of Puebla, Mexico. This activity has an estimated value of 625 million pesos per year and allows the participation of women in the regional economy. The use of high nitrogen fertilization on market roses, which surpasses $7000 \mathrm{~kg} \mathrm{ha}^{-1}$, is excessive and affects soil health and the economy of the producers. The objectives of this study were (1) to evaluate the current conditions of soil fertility in greenhouses that produce roses in Irrigation District 05 San Martín Texmelucan and (2) to study the effect of three sources of organic matter on the production, quality, and nutritional status of roses, and (3) to assess the physical condition of the treated soils. One hundred and twelve greenhouses located in four edaphoclimatic conditions were selected for the study. Soil samples were collected and their chemical properties evaluated to determine soil fertility. In a second experiment, three sources of organic matter (OM) were tested in one site: compost, cattle manure and chicken manure was 
applied on three varieties of rose: Anastasia, Selena and Latin. A modified completely randomized design was used. Number of stems/plant and stem length were measured, and analysis of variance and mean comparisons were performed. Soils had contrasting soil fertility properties. The quality of the roses increased with the three sources of OM three months after application but decreased in the ninth month. Chicken manure increased performance of the Anastasia variety and compost in the Selena variety. The OM sources increased the percentage of macroaggregates and the compost added to Anastasia and Selena produced the highest percentage. However, macronutrient concentrations in all rose plants were similar.

Index words: ornamentals, cut flower, soil health.

\section{INTRODUCCIÓN}

En el estado de Puebla existe una superficie cultivable de flores de aproximadamente 3600 ha, repartidas en 35 municipios, cuya producción anual alcanza un valor de producción de 625 millones de pesos (Milenio, 2009) $)^{1}$. La floricultura es una opción viable para los productores del campo, con mejores perspectivas que los cultivos tradicionales, debido a los mayores márgenes de rentabilidad que se pueden obtener de ella. Esta actividad ha ofrecido también la oportunidad de incorporar a la mujer a la economía regional, teniendo en cuenta que en la región del Estado donde se practica esta actividad se presenta alta migración (SAGARPA, 2013).

La producción de rosa para flor de corte en invernaderos se distingue por ser el sistema de cultivo más intensivo de la floricultura, ya que usa agua e insumos para fertilización en una elevada cantidad (Cabrera, 2003). La aplicación de fertilizantes es una de las principales actividades en la floricultura y ocupa un renglón importante en los costos del producto. $\mathrm{La}$ fertilización nitrogenada en rosa de corte presenta aplicaciones de nitrógeno elemental por arriba de $7000 \mathrm{~kg} \mathrm{ha}^{-1}$ anuales, lo que se considerada como excesivo. El uso indiscriminado de fertilizantes nitrogenados se asocia a la lixiviación de nitrógeno reportada en $3000 \mathrm{~kg} \mathrm{ha}^{-1}$ o mayor (Cabrera, 2005), lo que puede afectar la salud humana si el N llega al agua que se consume que alcanza niveles altos de nitratos.
La fertilización nitrogenada si no se hace de manera racional y con serias bases científicas (Castellanos et al., 2000) puede generar problemas indirectos de salinidad $\mathrm{y}$ desequilibrios nutricionales en el cultivo, que reducen la productividad y calidad de la flor cortada (Cabrera, 2005). Adicionalmente, contribuye a las emisiones de $\mathrm{N}_{2} \mathrm{O}$ aumentando la concentración de gases de efecto invernadero responsables del cambio climático.

La aplicación de nutrientes a rosa para flor de corte y otros cultivos en la región, se hacen sin prestar atención a las necesidades reales de éstos. En el manejo se incorporan escasamente aspectos basados en la fisiología del cultivo y la condición del suelo. Tampoco se toma en cuenta la historia previa de manejo y las condiciones climáticas. Si bien, se ha considerado el uso de fertirrigación en la rosa, se sabe que tal como se practica en California E.U.A., ha generado acumulación de sales (Cabrera y Perdomo, 2003) situación que afecta su producción y la calidad de los suelos. Por otra parte, existe una demanda creciente para usar productos inocuos que no deterioren el ambiente, como es el caso de distintos tipos de materia orgánica (Cruz et al., 2003).

Por lo anterior, los objetivos del presente trabajo fueron, (1) evaluar el estado actual de la fertilidad de los suelos en la zona de producción de rosa del DDR 05 San Martín Texmelucan. Se consideró como hipótesis que la adición de materia orgánica al suelo mejoraría su fertilidad. (2) Evaluar el efecto del aporte de tres tipos de MO en la producción, calidad y nutrición de tres variedades de rosa, así como en la condición física del suelo.

\section{MATERIALES Y MÉTODOS}

La zona de estudio se ubica en la región del estado de Puebla donde se ha desarrollado intensamente la floricultura, y comprende al distrito de desarrollo rural (DDR) 05 Cholula que forma parte del centro sur del Estado. Las zonas de producción de rosa en el DDR 05 son San Martín Texmelucan y el valle de Atlixco (SAGARPA, 2013). Este estudio se localizó en la primera de ellas (Figura 1).

De los municipios con más alta producción, Chiautzingo es el que tiene mayor número de invernaderos, representado con puntos verdes en la Figura 1.

${ }^{1}$ Milenio. 2009. Ya inició feria de la flor en Atlixco. http://impreso.milenio.com/node/8607067 (Consulta: enero 6, 2013). 


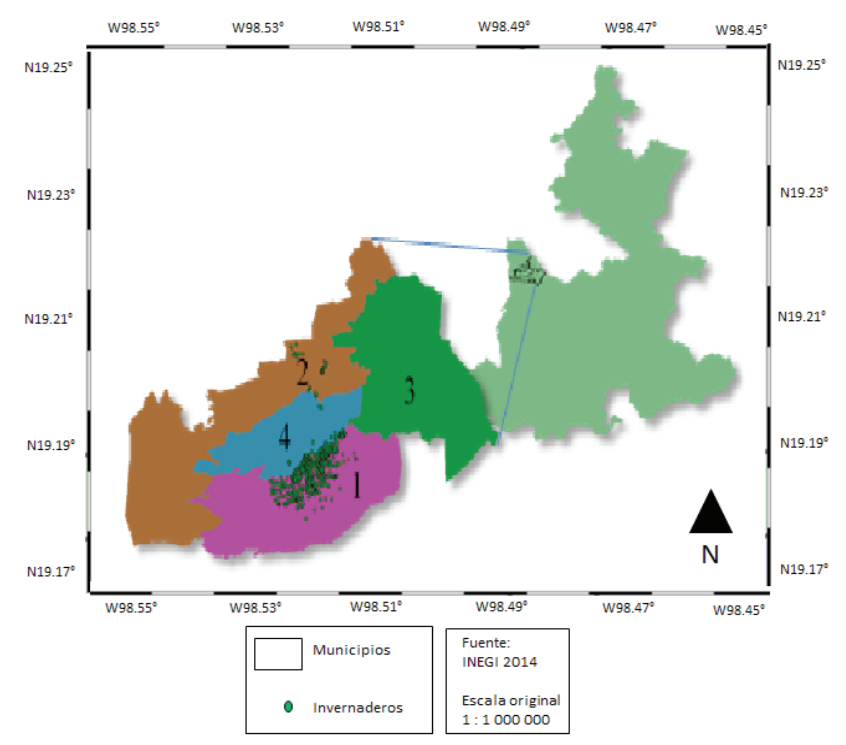

Figura 1. Zona de producción de rosa para flor de corte en los municipios del distrito de desarrollo rural (DDR) 05, San Martín Texmelucan: (1) San Lorenzo Chiautzingo, (2) San Salvador el Verde, (3) San Martín Texmelucan y (4) San Felipe Teotlalzingo (INEGI, 2014).

Con base en las cartas edafológicas (1:50000) e información climática de INEGI (2014) se identificaron cuatro principales unidades de suelo, Regosol y Cambisol éutrico compartiendo la misma unidad edafológica ( $\mathrm{Re}+\mathrm{Be} / 1)$ (S1), Fluvisol éutrico (Je/1) (S2), Cambisol dístrico y húmico (Bd+Bh) (S3), Regosol éutrico y luvisol (Re+1/1) (S4). Los climas que predominan son templado subhúmedo $C(w 2)(w)(\mathrm{C} 1)$ y semifrío $(C(E)(w 2)(w))(C 2)$. Con esta información y el software de sistemas de información geográfica ArcMAP se cruzó información para definir las cinco unidades edafoclimáticas existentes en la zona de estudio: S1C1, S2C1, S3C1, S4C1 y S1C2 (Figura 2). Los invernaderos productores de rosa de corte de la zona se georreferenciaron y se ubicaron en la unidad edafoclimática correspondiente, siendo éste el criterio de muestreo de suelos para la caracterización de la fertilidad del agrosistema.

\section{Muestreo de Suelo y Material Vegetal}

De un total de 668 invernaderos empadronados en la región se eligieron 112 al azar, los cuales quedaron localizados en las unidades edafoclimáticas de la siguiente manera: 39 en S1C1, 19 en la S2C1, 17 en la $\mathrm{S} 3 \mathrm{C} 1,8$ en la $\mathrm{S} 4 \mathrm{C} 1$ y 29 en la S1C2. La mayoría de los invernaderos se localiza en Regosol y Cambisol éutrico y en climas templado subhúmedo y semifrío (S1C1 y S1C2).

Para evaluar la fertilidad del suelo de los 112 invernaderos seleccionados se tomaron muestras entre febrero y marzo de 2014. Para definir el tamaño de la muestra se consideró lo señalado por Kogan y Herzog (1980) que definen el tamaño óptimo como aquel que permite un balance adecuado entre el costo del muestreo y la precisión deseada, lo que evita sobreestimación (sobre-gasto de recursos) o subestimación (precisión no adecuada). El tamaño de la muestra de cada unidad edafoclimática se determinó de manera estadística, se usó la fórmula de tamaño de muestra definida por Badii et al. (2011). Un segundo muestreo se realizó a los nueve meses después de haber establecido el experimento, solamente en uno de los invernaderos de la unidad edafoclimática $\mathrm{S} 1 \mathrm{C} 1(\mathrm{Re}+\mathrm{Be} / 1$ y C(w2)(w)), que presentó el mayor número de invernaderos. Este último se utilizó para evaluar el efecto del aporte de tres fuentes de $\mathrm{MO}$ en el rendimiento, calidad y condición nutrimental de la planta; así como la condición física del suelo.

En todos los casos, las muestras de suelo se tomaron en la base del surco de plantación de las rosas, a una profundidad de 0-15 cm. El muestreo se realizó en zigzag, se colectaron 15 submuestras que se mezclaron y

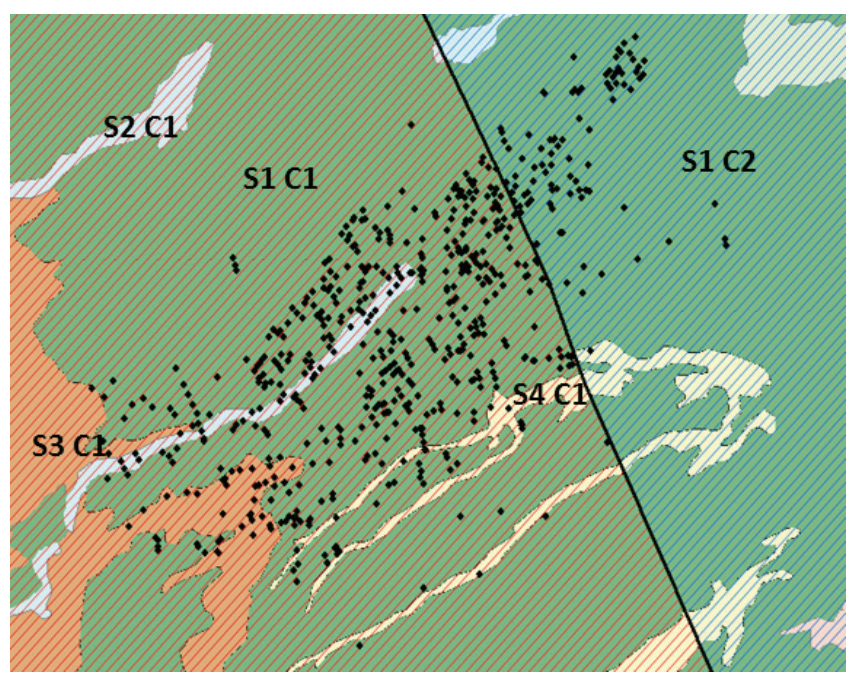

Figura 2. Unidades edafoclimáticas donde se ubica el agrosistema de producción de rosa en la ladera este del Iztaccíhuatl. (S1C1: $R e+B e / 1$ y $C(w 2)(w), S 2 C 1: J e / 1$ y C(w2) (w), S3C1: $B d+B h$ y $C(w 2)(w), S 4 C 1: R e+l / 1$ y C(w2)(w), S1C2: $\boldsymbol{R e}+\boldsymbol{B} \boldsymbol{e} / \mathbf{1}$ y $\boldsymbol{C}(\boldsymbol{E})(\boldsymbol{w} 2)(\boldsymbol{w}))$. (Los puntos negros corresponden a los invernaderos en la zona de estudio). 
homogeneizaron para obtener una muestra compuesta representativa (Castellanos et al., 2000). Las muestras compuestas se secaron a la sombra y se molieron hasta pasar por un tamiz de malla $2 \mathrm{~mm}$ para su posterior análisis.

Se realizaron dos muestreos de material vegetal, uno a los tres meses de haber establecido el experimento (mayo 2014) y el otro a los nueve meses (diciembre de 2014). Para el análisis de material vegetal se tomaron muestras compuestas foliares del primer par de hojas totalmente desarrolladas. El material se lavó con agua de grifo y clase I, se secó en estufa a $72{ }^{\circ} \mathrm{C}$ y se molió a malla $35(0.5 \mathrm{~mm})$ para su posterior análisis.

\section{Análisis de Suelo, Material Vegetal y Enmiendas Orgánicas}

A las muestras de suelo se les determinó el pH en una relación suelo:agua $(1: 2)$ por potenciometría, la materia orgánica (MO) con el método de Walkley y Black, el nitrógeno $(\mathrm{N})$ por el método semimicro Kjeldhal y el $\mathrm{P}_{\text {extraible }}\left(\mathrm{P}_{\text {ex }}\right)$ por el método de Olsen. Las bases $\mathrm{Na}, \mathrm{K}, \mathrm{Ca}$ y $\mathrm{Mg}$ intercambiables $\left(\mathrm{Na}_{\text {int, }}\right.$, $\mathrm{K}_{\text {int }}, \mathrm{Ca}_{\text {int }}, \mathrm{Mg}_{\text {int }}$ ) se extrajeron con $\mathrm{AcONH}_{4} 1 \mathrm{~N} \mathrm{pH} 7$ y los microelementos $\mathrm{Fe}, \mathrm{Zn}, \mathrm{Cu}, \mathrm{Mn}$ con DTPA. En el material vegetal se determinó el nitrógeno $(\mathrm{N})$ con el método semimicro Kjeldhal. Los elementos totales (P, $\mathrm{Na}, \mathrm{K}, \mathrm{Ca}, \mathrm{Mg}$ ) se obtuvieron con una digestión con ácido nítrico:perclórico y calentamiento a $210^{\circ} \mathrm{C}$. El Ca y $\mathrm{Mg}$ en suelo y material vegetal, así como el $\mathrm{Fe}, \mathrm{Cu}$, $\mathrm{Zn}$ y $\mathrm{Mn}$ en suelo se determinaron con espectroscopía de absorción atómica (EAA). Para determinar $\mathrm{K}$ en suelo y material vegetal se empleó la espectroscopía de emisión (EE). En todos los análisis de suelo se usaron las técnicas señaladas en la Norma (NOM-021SEMARNAT-2000). Las fuentes de materia orgánica usadas fueron compost comercial, estiércol de bovino y gallinaza. Éstas se analizaron para determinar el contenido de nitrógeno $(\mathrm{N})$ por el método semi-micro Kjeldhal, los elementos totales $(\mathrm{P}, \mathrm{K}, \mathrm{Ca}$ y $\mathrm{Mg}$ ) en un digerido nítrico:perclórico y la $\mathrm{MO}$ con el método de Walkley y Black.

Adicionalmente, en el invernadero de la unidad edafoclimática $\mathrm{S} 1 \mathrm{C} 1 \quad[\mathrm{Re}+\mathrm{Be} / 1$ y $\mathrm{C}(\mathrm{w} 2)(\mathrm{w})]$ se tomaron muestras para determinar la estabilidad de agregados a los nueve meses de establecido el experimento (diciembre del 2014). Se consideró que la evaluación de la estabilidad de los agregados en el suelo no sería significativa antes de ese tiempo.
De igual forma, ésta se hizo coincidir con el segundo muestreo de material vegetal. Se usaron cilindros de $5 \mathrm{~cm}$, las muestras se obtuvieron en la base del surco de plantación de las rosas. Las muestras inalteradas de suelo se obtuvieron antes y después de la aplicación de los materiales orgánicos (compost, estiércol bovino y gallinaza). Las muestras fueron secadas al aire, posteriormente el suelo se tamizó en una columna de tamices con aperturas de 4.75, 2, 1, 0.50, 0.25 y $0.05 \mathrm{~mm}$. La columna de tamices se sumergió en un cilindro con agua destilada, y se le imprimió un movimiento giratorio con una velocidad de $60 \mathrm{rpm}$ y se retiró del agua. La fracción del suelo retenida en cada uno de los tamices se secó en una estufa a $105^{\circ} \mathrm{C}$ durante 18 horas y se pesó.

\section{Evaluación de las Fuentes de Materia Orgánica (MO)}

Se seleccionaron tres fuentes de MO: compost comercial (T1), estiércol de bovino (T2) y gallinaza (T3), para evaluar su efecto en el rendimiento, calidad y nutrición de la planta; así como en la fertilidad química y física del suelo. Esta evaluación se llevó a cabo solamente en uno de los 112 invernaderos elegidos en la primera parte del estudio. Se seleccionó un invernadero en la unidad edafoclimática S1C1 $(\mathrm{Re}+\mathrm{Be} / 1$ y $\mathrm{C}(\mathrm{w} 2)(\mathrm{w}))$ que es la que presentó mayor número de invernaderos. Se evaluó el efecto de las fuentes de MO en tres variedades de rosa: Anastasia, Selena y Latín. Se definieron tres tratamientos. El criterio para definir cuánta enmienda se aplicaría en cada tratamiento fue mantener similar aporte de MO (Cuadro 1).

Los tratamientos se pusieron en unidades experimentales homogéneas, que consistieron en surcos de $16 \mathrm{~m}$ de largo $\times 50 \mathrm{~cm}$ de ancho (un surco para cada uno de los materiales evaluados). Cada tratamiento se replicó tres veces. Se generaron nueve unidades experimentales para cada variedad (tres fuentes de MO

Cuadro 1. Características y dosis de las enmiendas orgánicas utilizadas.

\begin{tabular}{|c|c|c|c|c|c|c|c|}
\hline & $\mathrm{N}$ & $\mathrm{P}$ & $\mathrm{K}$ & $\mathrm{Ca}$ & $\mathrm{Mg}$ & ${ }^{\dagger} \mathrm{MO}$ & Dosis \\
\hline & - & $\cdots$ & - - & $\%-$ & - & $-\cdots$ & $\mathrm{kg}$ \\
\hline Compost & 1.2 & 0.8 & 2.5 & 2.6 & 0.6 & 75.2 & 71.8 \\
\hline E. bovino & 1.4 & 0.33 & 1.9 & 1.3 & 0.2 & 54.07 & 99.8 \\
\hline Gallinaza & 0.2 & 1.3 & 2.5 & 3.1 & 0.5 & 26.34 & 117.1 \\
\hline
\end{tabular}

Materia orgánica, ${ }^{\ddagger}$ Dosis del producto por unidad experimental. 
$\times$ tres repeticiones). Considerando las tres variedades de rosas se tuvo un total de 27 unidades experimentales. Como las plantaciones se hacen en doble hilera sobre la base del surco, el total de plantas por unidad experimental fue aproximadamente de 90. El diseño experimental fue completamente al azar modificado, se consideraron las tres variedades de rosa y las tres fuentes de MO.

La primera evaluación de las plantas de rosa se realizó tres meses después de haber impuesto los tratamientos (6 al 10 de mayo del 2014). Las variables evaluadas fueron: rendimiento (número de tallos florares) y calidad (longitud de tallos florales). Para evaluar la variable de calidad se estableció un criterio arbitrario, definido de acuerdo con la experiencia de lo que el mercado reclama. Las clases de calidad se definieron de acuerdo a la longitud de tallo: calidad baja menor a $30 \mathrm{~cm}$, calidad media entre 30 y $50 \mathrm{~cm}$ y calidad alta mayor a $50 \mathrm{~cm}$. La segunda evaluación de las plantas de rosa se realizó nueve meses después de la aplicación de los tratamientos (5 al 12 de diciembre del 2014). Las variables evaluadas fueron: rendimiento y calidad (similar a la primera evaluación) y adicionalmente el contenido nutrimental en la planta.
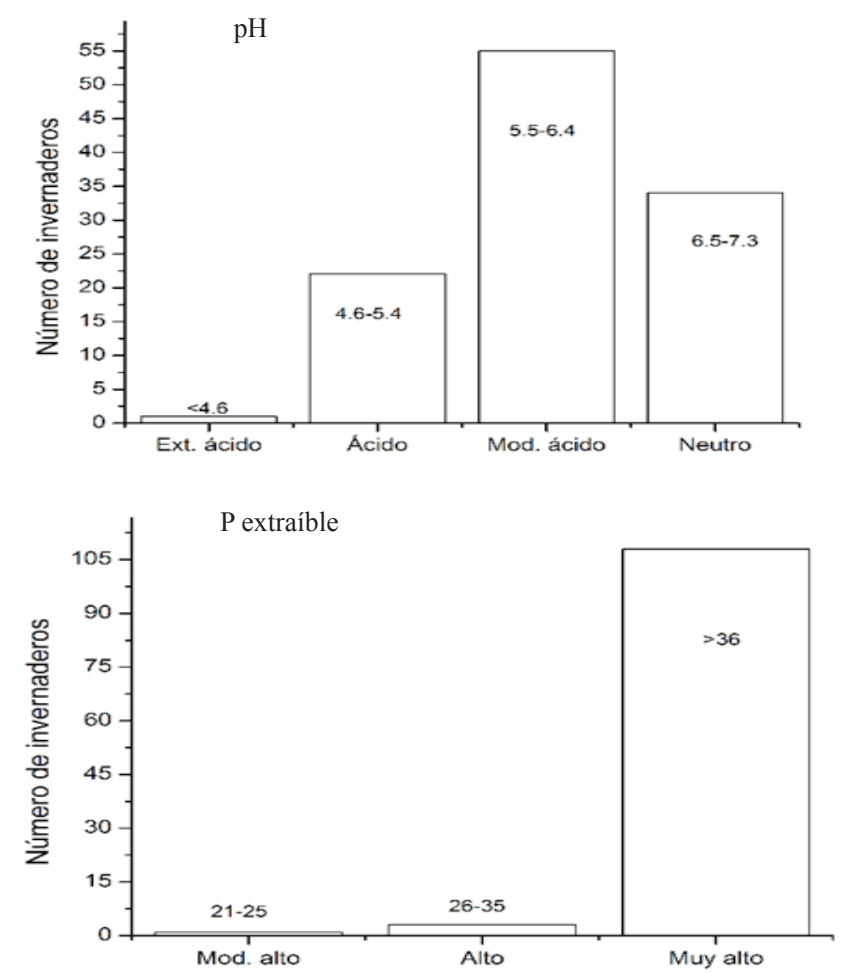

La interpretación del contenido nutrimental en hojas de las variedades evaluadas se llevó a cabo considerando los niveles reportados por Benton et al., (1991) y por Urbina $(2000)^{2}$ que corresponden a los niveles nutrimentales en hojas pentafoliadas de rosal cuando el botón muestra color. Si bien, esta etapa del desarrollo de la planta no corresponde a la de las hojas muestreadas, fueron los únicos datos disponibles que se podían considerar para dicha interpretación.

Con los datos obtenidos de rendimiento, calidad de producción y concentración de nutrimentos en planta, se realizaron los análisis de varianza y pruebas de comparaciones múltiples de medias de acuerdo el método de Tukey con nivel de significancia alfa $=0.05$.

\section{RESULTADOS Y DISCUSIÓN}

La evaluación de las propiedades químicas de muestras de suelo provenientes de 112 invernaderos de la zona de estudio definió el estado de fertilidad del suelo del agroecosistema rosa. La mayor variabilidad entre los suelos se observó en los valores de pH y los contenidos de $\mathrm{MO}, \mathrm{P}_{\text {ext }} \mathrm{y} \mathrm{K}_{\text {int }}$ (Figura 3).

El pH de más de la mitad de las muestras fue de
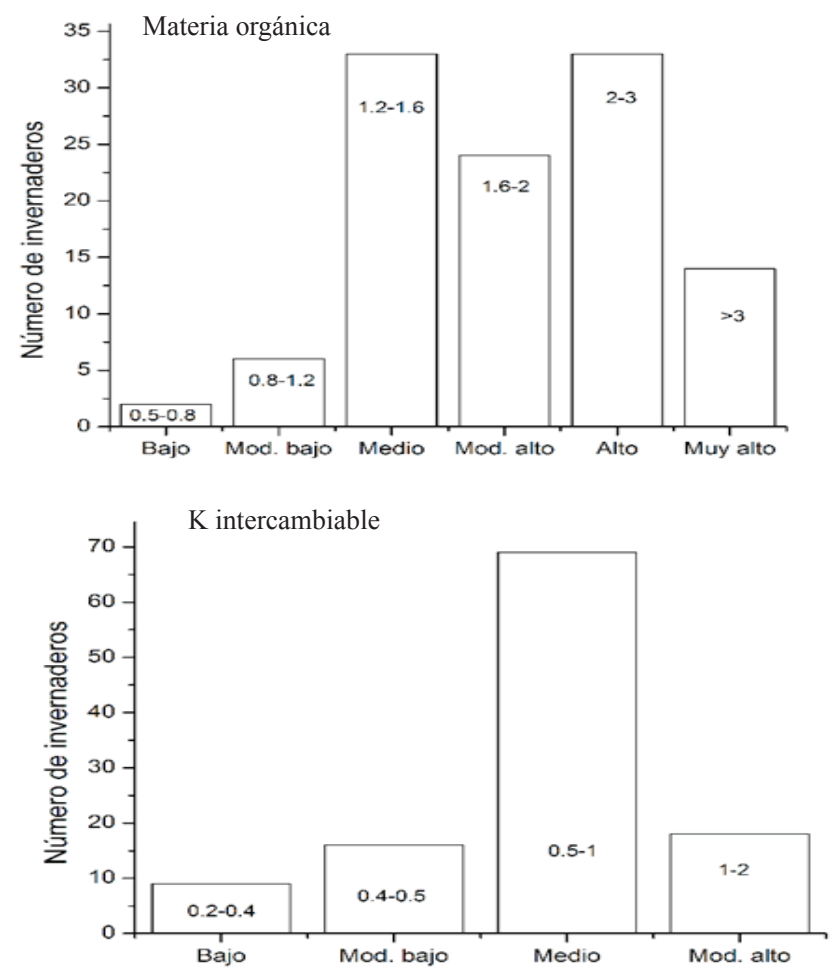

Figura 3. Características químicas de suelos del agrosistema rosa en la zona de producción de San Martín Texmelucan del DDR 05,

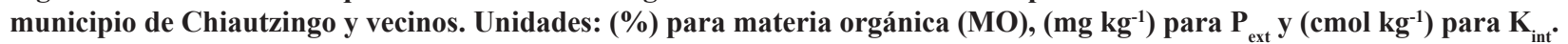

${ }^{2}$ Urbina-Sánchez, E. 2000. Uso de diferentes tipos de vermicomposta como fertilizante orgánico en el cultivo del rosal. Tesis de Maestría en Ciencias en Horticultura. Departamento de Fitotecnia. UACh. Chapingo, México. 
moderadamente ácido a neutro $(\mathrm{pH} 5.5$ a 7.3). Estos valores son muy cercanos al rango 6.4 a 7.5 considerado por Heitz (1994) como adecuados para la rosa. Los niveles de MO no representan un problema serio en los suelos, la mayoría presentó niveles de MO de medios a muy altos (de 1.2\% a más de 3\%) (Figura 3 ). Sin embargo, destacan las elevadas concentraciones de $\mathrm{P}_{\text {ext }}$ (Olsen) mayores a $36 \mathrm{mg} \mathrm{kg}^{-1}$, en más del $96 \%$ de las muestras evaluadas. Castellanos et al. (2000) reporta estos niveles como concentraciones excesivas (Figura 3). Los niveles encontrados en el suelo de $\mathrm{P}_{\text {ext }}$ evidencian el manejo empírico que actualmente practican los productores de la zona, que no presta atención a los niveles existentes de elementos químicos en los suelos ni a los requerimientos de éstos por el cultivo. De continuar aplicando en exceso este elemento, esto afectará la condición económica de los productores y la salud del suelo, además, representa un costo económico y ambiental que no es justificable. Una proporción importante de los suelos de los invernaderos presentaron concentraciones de $\mathrm{K}_{\text {int }}$ entre 0.5 y $1 \mathrm{cmol} \mathrm{kg}^{-1}$, niveles considerados medios y ligeramente altos. Otras bases de intercambio como $\mathrm{Ca}$ $\left(1.2-11.2 \mathrm{cmol} \mathrm{kg}^{-1}\right)$ y $\mathrm{Mg}\left(0.2-5 \mathrm{cmol} \mathrm{kg}^{-1}\right)$ presentaron concentraciones que se ubican en los rangos de muy bajo a medio para $\mathrm{Ca}$ y de bajo a alto para $\mathrm{Mg}$. Los niveles de microelementos variaron de moderadamente altos a muy altos para $\mathrm{Zn}$ y $\mathrm{Mn}\left(1.1\right.$ a $17 \mathrm{mg} \mathrm{kg}^{-1}$ ) y fueron muy altos para $\mathrm{Fe}$ (mayores a $\left.50 \mathrm{mg} \mathrm{kg}^{-1}\right)$ y $\mathrm{Cu}(1.2 \mathrm{a}$ $14.2 \mathrm{mg} \mathrm{kg}^{-1}$ ) (Castellanos et al., 2000).

\section{Evaluaciones en Planta (Rendimiento, Calidad y Contenido Nutrimental)}

Como resultado a la adición de las tres fuentes de MO evaluadas, no se observó un efecto estadísticamente significativo en rendimiento (tallos por planta) para las variedades Selena y Latin a los tres meses de haber sido establecido el experimento (mayo 2013) (Figura 4a). En la variedad Anastasia si hubo efecto por la fuente de MO adicionada, la gallinaza (T3) produjo el mayor rendimiento (tallos por planta) (Figura 4a). Sin embargo, nueve meses después de establecido el experimento (diciembre 2014), fue la variedad Selena la única que mostró efecto estadísticamente significativo por la fuente de MO adicionada al presentar mayor rendimiento (tallos por planta) con aplicación de compost (T1) (Figura 4b). El rendimiento de la variedad Latin no mostró efecto estadísticamente significativo debido a las fuentes de $\mathrm{MO}$ a los tres y nueve meses (Figura 4b).
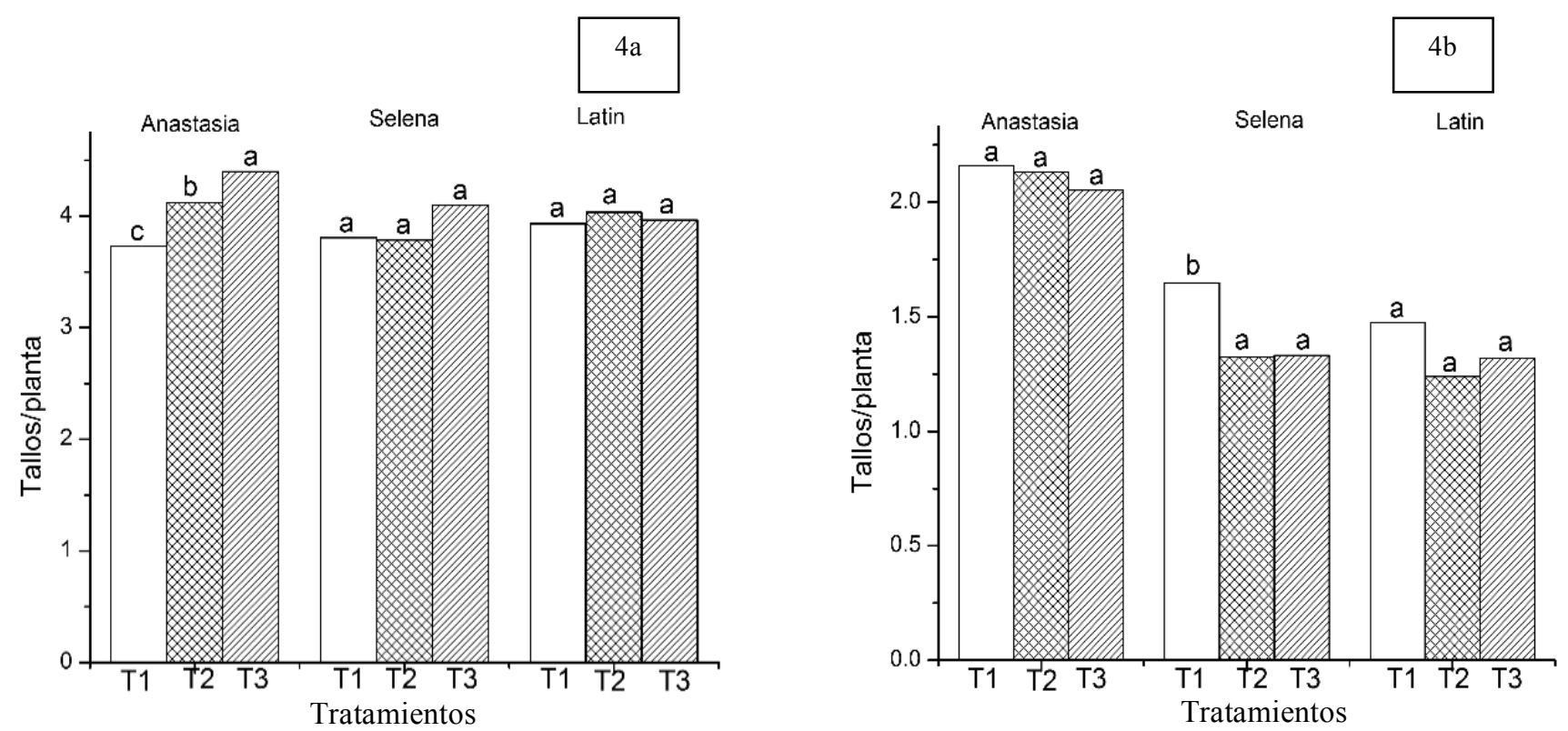

Figura 4. Rendimiento (tallos por planta) de las variedades Anastasia, Selena y Latin con diferentes adiciones de materia orgánica al suelo: compost (T1), estiércol de bovino (T2) y gallinaza (T3), a los 3 meses (4a) y 9 meses (4b) después de establecido el experimento. Medias con letra igual indican diferencias no significativas de acuerdo a la prueba de Tukey (al 5\%). 
La mayor calidad (mayor número de tallos de 30 a $50 \mathrm{~cm}$ y mayores a $50 \mathrm{~cm}$ ) se obtuvo a los tres meses de haber sido establecido el experimento (Figura 5a). Las tres variedades de rosas (Anastasia, Selena y Latin) presentaron más de 150 tallos con las tres fuentes de MO adicionada, compost (T1), estiércol bovino (T2) y gallinaza (T3). En las variedades Anastasia y Selena el efecto sobre la calidad fue mayor con la aplicación de gallinaza (T3) y estiércol de bovino (T2). En la variedad Latin, no se observó efecto significativo en la cantidad de tallos por la fuente de MO (Figura 5a). La calidad de las tres variedades de rosas disminuyó a los nueve meses de establecido el experimento, solamente se obtuvieron 120 o menos tallos de 30 a $50 \mathrm{~cm}$ y mayores a $50 \mathrm{~cm}$ (Figura $5 \mathrm{~b}$ ).

De las tres variedades de rosa, la Anastasia fue la de mayor calidad (Figura 5b).

El contenido nutrimental en las variedades Anastasia, Selena y Latin no mostró diferencia estadísticamente significativa debido a las fuentes de aporte de la MO a los nueve meses de establecido el experimento (Cuadro 2). Las concentraciones de $\mathrm{N}, \mathrm{P}$ y $\mathrm{Mg}$ fueron más bajas que el rango adecuado
Cuadro 2. Contenido nutrimental en hojas de las variedades de rosa Anastasia, Selena y Latin a los nueve meses de establecido el experimento.

\begin{tabular}{|c|c|c|c|c|c|}
\hline Tratamiento & $\mathrm{N}$ & $\mathrm{P}$ & $\mathrm{K}$ & $\mathrm{Ca}$ & $\mathrm{Mg}$ \\
\hline & \multirow{2}{*}{\multicolumn{5}{|c|}{$\begin{array}{r}-\ldots \\
\quad \ldots+\ldots \\
\quad-\ldots \text { Anastasia }\end{array}$}} \\
\hline & & & & & \\
\hline $\mathrm{T} 1$ & $2.4 \mathrm{a}$ & $0.2 \mathrm{a}$ & $2.1 \mathrm{a}$ & $1.4 \mathrm{a}$ & $0.2 \mathrm{a}$ \\
\hline $\mathrm{T} 2$ & $2.4 \mathrm{a}$ & $0.2 \mathrm{a}$ & $2.1 \mathrm{a}$ & $1.3 \mathrm{a}$ & $0.2 \mathrm{a}$ \\
\hline \multirow[t]{2}{*}{$\mathrm{T} 3$} & $2.1 \mathrm{a}$ & $0.2 \mathrm{a}$ & $2.0 \mathrm{a}$ & $1.2 \mathrm{a}$ & $0.2 \mathrm{a}$ \\
\hline & \multicolumn{5}{|c|}{ Selena } \\
\hline $\mathrm{T} 1$ & $2.2 \mathrm{a}$ & $0.2 \mathrm{a}$ & $2.1 \mathrm{a}$ & $1.3 \mathrm{a}$ & $0.2 \mathrm{a}$ \\
\hline $\mathrm{T} 2$ & $2.3 \mathrm{a}$ & $0.2 \mathrm{a}$ & $2.1 \mathrm{a}$ & $1.3 \mathrm{a}$ & $0.2 \mathrm{a}$ \\
\hline \multirow[t]{2}{*}{$\mathrm{T} 3$} & $2.3 \mathrm{a}$ & $0.2 \mathrm{a}$ & $2 \mathrm{a}$ & $1.4 \mathrm{a}$ & $0.3 \mathrm{a}$ \\
\hline & \multicolumn{5}{|c|}{ Latin } \\
\hline $\mathrm{T} 1$ & $2.2 \mathrm{a}$ & $0.2 \mathrm{a}$ & $1.8 \mathrm{a}$ & $1.1 \mathrm{a}$ & $0.3 \mathrm{a}$ \\
\hline $\mathrm{T} 2$ & $2.2 \mathrm{a}$ & $0.3 \mathrm{a}$ & $1.8 \mathrm{a}$ & $0.9 \mathrm{ab}$ & $0.2 \mathrm{a}$ \\
\hline T3 & $2.1 \mathrm{a}$ & $0.2 \mathrm{a}$ & $1.5 \mathrm{a}$ & $1.2 \mathrm{a}$ & $0.2 \mathrm{a}$ \\
\hline
\end{tabular}

T1: compost ; T2: estiércol de bovino ; T3: gallinaza. Medias con letra igual indican diferencias no significativas de acuerdo a la prueba de Tukey (al 5\%).
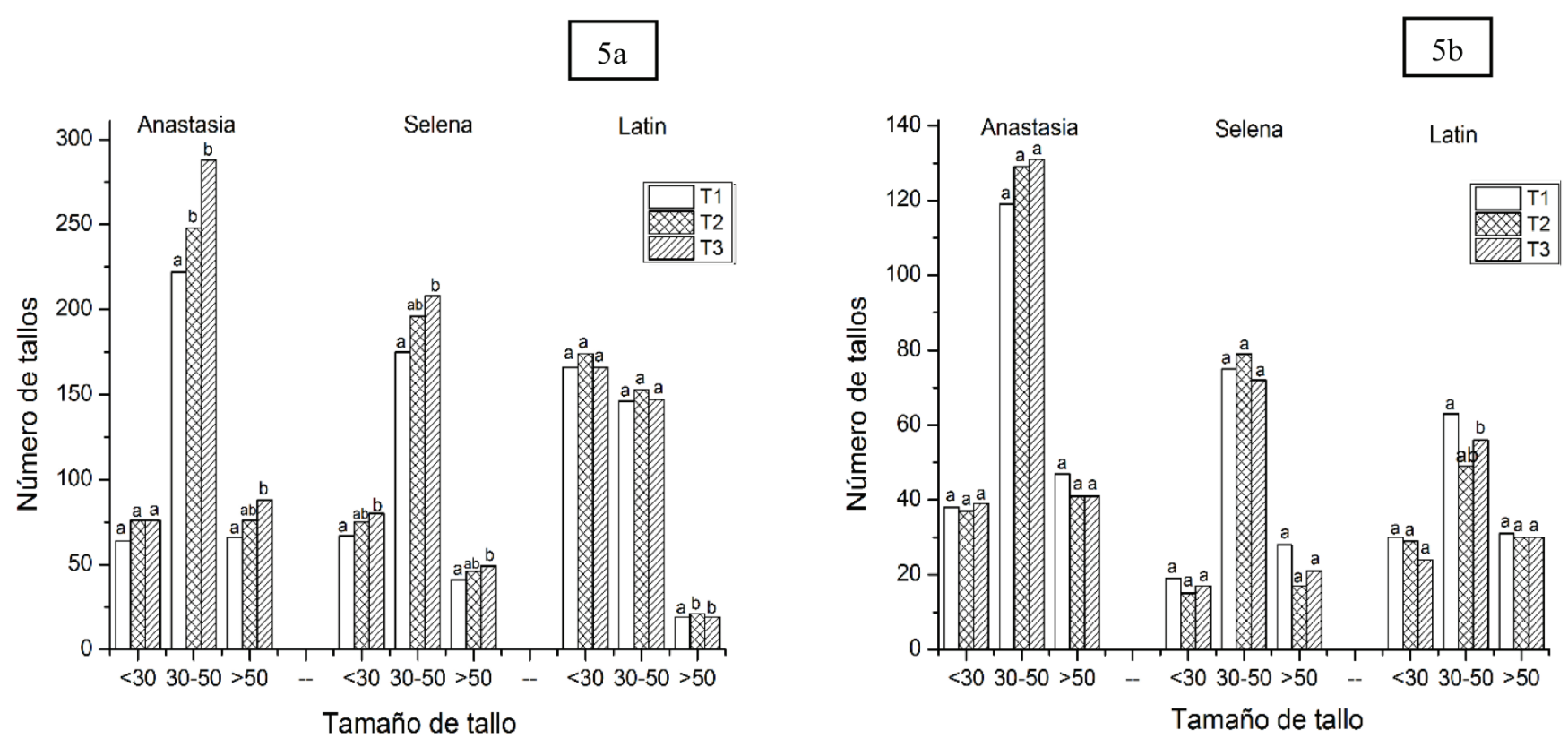

Figura 5. Calidad (tamaño de tallos) de las variedades Anastasia, Selena y Latin por adición de materia orgánica: compost (T1), estiércol de bovino (T2) y gallinaza (T3) a los 3 meses (5a) y los 9 meses (5b) después de establecido el experimento. Medias con letra igual indican diferencias no significativas de acuerdo a la prueba de Tukey (al 5\%). 
reportado por Suárez $(2006)^{3}$ para rosa. El N con una concentración menor al $2 \%$ se consideró como deficiente. No se esperaba este resultado ya que el aporte de este elemento a la parcela muestreada fue $150 \mathrm{~kg}$ en una combinación de las formas orgánica (MO) e inorgánica (fertilizantes). Este resultado se explicaría por el exceso de agua que se aplica en el riego rodado usado en los invernaderos. Esto habría conducido a la lixiviación del nitrógeno a capas de suelo más profundas lo que impide la toma del nutriente para el desarrollo de la planta.

De seguirse dando el mismo manejo de riego podrían ocasionarse problemas de contaminación de las napas freáticas. Además, esto también limitará el rendimiento y calidad de la rosa. El P presentó concentraciones consideradas como óptimas (Suárez,
2006). Los niveles de $\mathrm{K}$ y $\mathrm{Ca}$ encontrados en las muestras foliares se ubicaron dentro de los rangos de bajo a suficiente. Solo se observaron ligeras diferencias significativas en los niveles de $\mathrm{Ca}$ en la variedad Latin. El Mg presentó mayor variabilidad, deficiente, baja y suficiente en su concentración.

\section{Efecto de la Aplicación de Materia Orgánica a la Condición Física del Suelo}

El efecto de la condición física del suelo por el aporte de distintos tipos de materia orgánica ( $\mathrm{T} 1$, T2 y T3), se observó claramente en las variedades Anastasia y Selena, y solo ligeramente en la variedad Latin. Los porcentajes más altos de macroagregados (0.25-1 mm) se observaron después de la aplicación
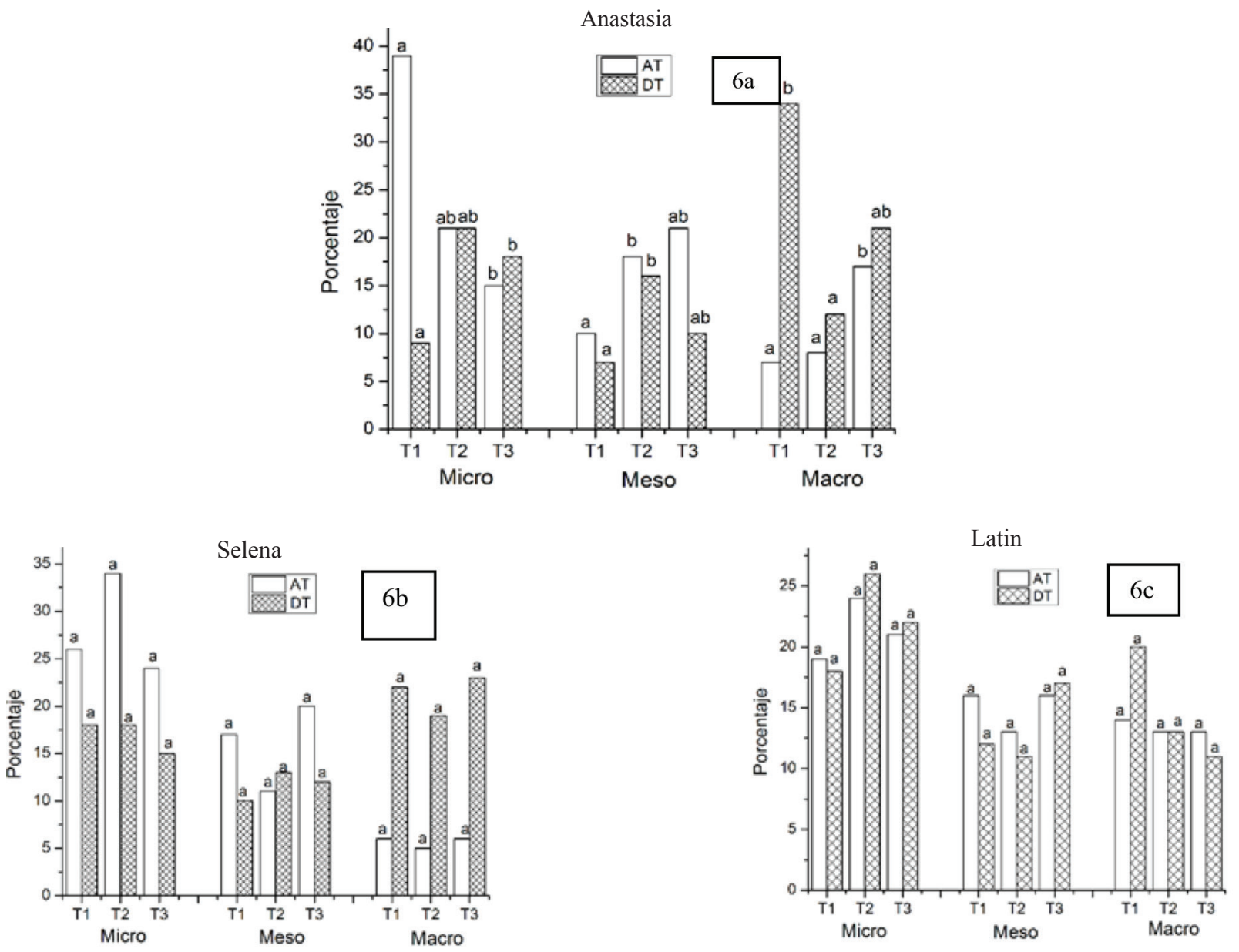

Figura 6. Distribución de micro, meso, y macroagregados de suelo antes (AT) y después (DT) de la aplicación de materia orgánica: compost (T1), estiércol de bovino (T2), gallinaza (T3) a las tres variedades de rosa Anastacia (6a), Selena (6b) y Latin (6c). Letras diferentes en sentido vertical muestran tratamientos con diferencias significativas. *Nivel de significancia $\alpha=0.05$.

${ }^{3}$ Suárez Santana, Juan Antonio. 2006. Fertilidad de los suelos y recomendación de fertilización en el Rosal (Rosa sp.) de Coatepec Harinas, Méx. Tesis Profesional de Licenciatura. Departamento de Suelos. UACh. Chapingo, México. 
de compost (T1), en las tres variedades (Figura 6). El compost es un material más evolucionado que los estiércoles, y contiene moléculas más estables que sirven para enlazar los microagregados, los cuales pasan a formar parte de los meso y macroagregados, generando una mejor estructura del suelo (Six et al., 2002). En la variedad Selena, la adición de estiércol de bovino (T2) y gallinaza (T3) (Figura 6b) también promovió la formación de macroagregados, aún cuando el análisis estadístico indicó que no hubo diferencias significativas. El aumento de la macroporosidad permite una mayor infiltración de agua y porosidad lo que beneficia el desarrollo de raíces. El porcentaje de mesoagregados no presentó diferencias significativas debido a los tipos de aporte de MO. Ni tampoco los porcentajes de macro y microagregados en el suelo tanto antes como después de adicionar estiércol bovino (T2) y gallinaza (T3) en las tres variedades de rosas (Figura 6).

Los microagregados en el suelo predominaron antes de aplicar compost (T1) a la variedad Anastacia (Figura 6a), y las tres fuentes de MO a la variedad Selena (Figura 6b). Esto se explica por el efecto de rompimiento de los agregados de suelo asociado a las labores de deshierbe y al sistema de riego rodado. Con ello la consecuente pérdida de materia orgánica del suelo (MOS) requerida para la agregación y cohesión entre las partículas del suelo (Tisdall y Oades 1982, Six et al., 2002).

\section{CONCLUSIONES}

- Los suelos de los invernaderos del agrosistema rosa (Rosa spp.) ubicados en la ladera este del Iztaccíhuatl presentaron características químicas y condiciones de fertilidad contrastantes, producto de una variabilidad edafoclimática.

- La fuente de materia orgánica (MO) adicionada al suelo y el tiempo transcurrido después del establecimiento de las plantas tuvo un efecto en el rendimiento de rosa (tallos/planta). La gallinaza aumentó el rendimiento en la variedad Anastasia a los tres meses y el compost en la variedad Selena a los nueve meses después del establecimiento del experimento.

- La calidad (longitud de tallos florales) de las tres variedades de rosas aumentó como producto del aporte de las tres fuentes de MO al suelo, tres meses después de establecido el experimento. La gallinaza y el estiércol de bovino promovieron una mayor calidad en las variedades Anastasia y Selena. La calidad de las tres variedades de rosas disminuyó a los nueve meses de instalado el experimento.

- Los niveles de macroelementos en hoja fueron similares con las tres fuentes de MO a los nueve meses de establecido el experimento. Los niveles bajos de $\mathrm{N}$ en suelo y muestras foliares indican la necesidad de atender el manejo y fertilización de este elemento.

- Las tres fuentes de MO adicionada mejoraron la condición física del suelo al aumentar el porcentaje de macroagregados en los suelos de las tres variedades de rosas. La adición de compost condujo a un mayor porcentaje de macroagregados en el suelo con las variedades Anastasia y Selena.

\section{LITERATURA CITADA}

Badii-Zabeh, M. H., A. Guillen-Gaytan, E. Cerna-Chavez y J. R. Valenzuela-Garcia. 2011. Nociones introductorias de muestreo estadístico. Daena: Int. J. Good Consci. 6: 89-105.

Benton Jones, J. Jr., B. Wolf, and H. A. Mills. 1991. Plant analysis handbook. A practical sampling, preparation, analysis, and interpretation guide. Micro-Macro Publishing, Inc. Athens, GA, USA.

Cabrera, R. I. 2003. Demarcating salinity tolerance in greenhouse roses. Acta Hortic. 609: 51-57.

Cabrera, R. I. 2005. Salinity tolerance in greenhouse roses: Whole plant biomass and nutrient partitioning. International Cut Flower Growers Assn. Bulletin, February Issue: 15-21.

Cabrera, R. I. and P. Perdomo. 2003. Reassessing the salinity tolerance of greenhouse roses under soilless production conditions. HortScience 38: 533-536.

Castellanos, J. Z., J. X. Uvalle-Bueno y A. Aguilar-Santelises. 2000. Manual de interpretación de análisis de suelos y aguas. Colección INCAPA. México.

Cruz-Rodríguez, V., V. C. de Almeida-Theodoro, I. Francisco de Andrade, A. Inácio-Neto, V. do Nascimento-Rodrigues, e F. Villa-Alves. 2003. Produção de minhocas e composição mineral do vermicomposto e das fezes procedentes de bubalinos e bovinos. Ciênc. Agrotec. 27: 1409-1418.

Heitz, H. 1994. Rosas. Asesoramiento técnico sobre su plantación, cultivo, poda y reproducción. Everest. Madrid, España.

INEGI (Instituto Nacional de Estadística y Geografía). 2014. Censo general de población y vivienda. Instituto de Estadística, Geografía e Informática. www. inegi.org.mx. (Consulta: febrero 25, 2014).

Kogan, M. and D. C. Herzog. 1980. Sampling methods on soybean entomology. Springer-Varlag. New York, NY, USA.

Norma Oficial Mexicana NOM-021-SEMARNAT-2000. 2000. Que establece las especificaciones de fertilidad, salinidad y clasificación de suelos. Estudios, muestreo y análisis. http://dof. gob.mx/nota_detalle.php?codigo $=717582 \&$ fecha $=31 / 12 / 2002$. (Consulta: marzo 15, 2013). 
SAGARPA (Secretaría de Agricultura, Ganadería, Desarrollo Rural, Pesca y Alimentación). 2013. Actualización de la caracterización de la cadena productiva de ornamentales: Gladiola y rosa (flor de corte). Equipo de Asistencia Técnica del DDR 05 Cholula. Puebla, México.
Six, J., C. Feller, K. Denef, S. Ogle, J. C. de Moraes Sa, and A. Albrecht. 2002. Soil organic matter, biota and aggregation in temperate and tropical soils-effects of no tillage. Agronomie 22: $755-775$.

Tisdall, J. M. and J. M. Oades. 1982. Organic matter and watestable aggregates in soils. J. Soil Sci. 62: 141-163. 